\title{
Uma análise do preparo e enfrentamento da equipe de enfermagem em Manaus para com o atendimento ao paciente em crise psicótica
}

\author{
An analysis of the preparation and coping of the nursing team in Manaus for care to the patient in \\ psychotic crisis
}

\author{
Ana Crisllen Monteiro Sales ${ }^{1}$, Ayrton Brandão da $\operatorname{Silva}^{1}{ }^{\ominus}$, Diana Karen Sales da Silva ${ }^{\bullet}$, Igor \\ Klisman da Silva Lima $^{1}{ }^{\ominus}$, Half Adriel Simplício Araújo ${ }^{\oplus}$, Leandro Silva Pimentel ${ }^{2}$
}

${ }^{1}$ Enfermagem da Faculdade Estácio do Amazonas, Manaus, Amazonas, Brasil. ²Docente do Curso de Enfermagem da Faculdade Estácio do Amazonas, Manaus, Amazonas, Brasil. *Autor para correspondência. E-mail: enfermeiro.leandropimentel@gmail.com

\begin{abstract}
Resumo: Introdução: Estudo centrado no atendimento primário realizado em pacientes com crise psicóticas em hospitais em Manaus, com objetivo de caracterizar e analisar o enfrentamento da enfermagem perante ao atendimento emergencial a pacientes com crises psicóticas. Métodos: Realizou-se uma pesquisa online quantitativa qualitativa exploratória com 38 participantes atuantes na área da Enfermagem. Resultados e discussão: Baseado na análise de pesquisa onde predominaram participantes do gênero feminino, destacou-se a insegurança e falta de habilidade diante ao atendimento de crise psicótica. Conclusão: O bom atendimento primário é de extrema importância para o sistema hospitalar, evidenciouse a necessidade de uma educação continuada dando condições e preparo perante ao atendimento emergencial psiquiátrico, incentivando um atendimento cordial e humanizado ao paciente com transtorno mental.
\end{abstract}

Palavras-chave: assistência de enfermagem, atendimento primário, paciente em crise psicótica.

\begin{abstract}
Introduction: Study focused on primary care performed in patients with psychotic crises in hospitals in Manaus with the objective of characterize and analyze nursing coping with emergency care for patients with psychotic crises. Methods: A qualitative exploratory quantitative field research was carried out with 38 participants working in the field of Nursing. Results: Based on research analysis where female participants predominated, insecurity and lack of ability in the face of psychotic crisis stood out. Conclusion: Good primary care is of utmost importance for the hospital system, the need for continuing education was evidenced, providing conditions and preparation for emergency psychiatric care, encouraging cordial and humanized care for patients with mental disorders.
\end{abstract}

Keywords: nursing assistance, primary care, patient in psychotic crisis.

\section{Introdução}

A Atenção Básica tem como um de seus princípios possibilitar o primeiro acesso das pessoas ao sistema de Saúde, inclusive daquelas que demandam um cuidado em saúde mental. Podemos dizer que o cuidado em saúde mental na Atenção Básica é bastante estratégico pela facilidade de acesso das equipes aos usuários e vice-versa. Por estas características, é comum que os profissionais de Saúde se encontrem a todo o momento com pacientes em situação de sofrimento psíquico. No entanto, apesar de sua importância, a realização de práticas em saúde mental na Atenção Básica suscita muitas dúvidas, curiosidades e receios nos profissionais de Saúde (Ministério da Saúde, 2013).

O sentimento de se sentir incapaz de se aproximar de um paciente em crise psicótica é bastante comum, tanto para o profissional de Saúde quanto para a população em geral. Esta convicção se deve desde o período de isolamento e exclusão no qual estás pessoas vem sendo submetidas a vivenciar a experiência com a "loucura" (Kabbe et al., 2014).

Outro elemento a ser levado em consideração na superação dessa aparente limitação, é o reconhecimento por parte do profissional, do intenso sofrimento vivenciado pelo paciente. É necessário ter a capacidade de analisar e garantir o bom cuidado assistencial e continuado, a crise deve ser compreendida como parte do cotidiano dos pacientes que seguem constantemente com situações que forjam desorganização em sua vida (Kondo et al., 2014). 
O perfil epidemiológico das emergências psiquiátricas (EP) apresentam mudanças constantes e significativas nos últimos anos correlacionado com a reforma psiquiátrica, mudando assim a rotina de toda a equipe e serviços de saúde. Por conta disto, atualmente as situações de crise psicótica são inicialmente atendidas em serviços de emergência, tendo destaque aos hospitais gerais (Ministério da Saúde, 2015).

É necessário que a equipe de enfermagem esteja preparada perante a estes atendimentos, obtendo não só de uma preparação técnica como emocional para promover cuidados aos portadores de transtornos mentais e aos seus familiares, sabendo o seu espaço de atuação e construindo uma conduta de atendimento mais eficaz perante a uma emergência psiquiátrica. Perante aos fatos e de extrema necessidade que a enfermagem tenha total conhecimento das condutas a serem realizadas, o preparo e a disponibilidade emocional relacionadas as crises psicóticas admitidas, colocando em evidencia a atual organização da assistência no contexto da saúde mental (Costa et al., 2016).

\section{Métodos}

Trata-se de um estudo quantitativo, qualitativo, descritivo e transversal realizado através de uma pesquisa online, usando como suporte de ferramenta a plataforma www.onlinepesquisa.com, onde foi elaborado um questionário focado em analisar as respostas coletadas pelos profissionais da área de saúde. A amostra foi composta por 38 trabalhadores da área da saúde atuantes e estudantes da área com objetivo de realizar uma análise do preparo e enfrentamento da equipe de enfermagem em Manaus perante ao atendimento ao paciente em crise psicótica. A coleta de dados foi realizada entre os meses de fevereiro e março de 2020.

Esta pesquisa foi submetida a uma avaliação livre, onde cada profissional respondia de maneira individual um questionário que foi realizado em três etapas: a primeira onde os participantes respondiam perguntas objetivas sobre gênero, atuação profissional e tempo de atuação. Na segunda foram apresentadas por questões polar, cujas respostas esperadas eram "sim ou não". Na terceira e última foram usadas questões subjetivas no qual foram respondidas conforme perspectiva individual de cada participante perante ao seu conhecimento, enfrentamento e necessidade.

Como critério de inclusão na pesquisa, foram estabelecidos foco nos profissionais da saúde, Enfermeiro (a), Técnico de enfermagem, Acadêmicos de Enfermagem e Docentes, além desta condição de ser entrevistado cada participante concordou em participar da pesquisa onde o anonimato foi assegurado.

Foram observadas diferentes opiniões, assim dividindo a pesquisa em profissionais prontos para receber pacientes em crises psicóticas e profissionais que não se sentem prontos para atuar na linha de frente com o atendimento em decorrência ao paciente em crise psicótica.

\section{Resultados}

A coleta e análise realizada por meio do questionário semiestruturado, foi submetida a criação de um perfil de variáveis geral dos trabalhadores participantes da pesquisa. Sobre o enfrentamento e preparo da equipe perante ao atendimento em emergências psiquiátricas foram registradas em formato de tabela facilitando a visualização e analise dos resultados.

Tabela 1. Variáveis do perfil geral dos trabalhadores da enfermagem.

\begin{tabular}{ccc}
\hline Variáveis & $\mathrm{N}^{\mathrm{o}}$ & $\%$ \\
\hline Gênero & & 75 \\
Feminino & 25 & 15 \\
Masculino & 13 & $\%$ \\
\hline Atuação Profissional & $\mathrm{N}^{\mathrm{o}}$ & 35 \\
\hline Técnico de Enfermagem & 11 & 55 \\
Enfermeiro & 17 & 10 \\
Outros & 10 & $\%$ \\
\hline Tempo de atuação profissional & $\mathrm{N}^{\mathrm{o}}$ & 7 \\
\hline $1-3$ & 7 & 7 \\
\\
$4-5$ & 7 & 86 \\
\hline Total & 24 & 100
\end{tabular}

Conforme a tabela 1, observa-se que há predomínio do gênero feminino e Enfermeiros, com tempo de atuação variado entre 1 a 10 anos, onde a média de 6 a 10 anos no tempo de atuação predomina. 
Uma análise do preparo e enfrentamento da equipe de enfermagem em Manaus para com o atendimento ao paciente em crise

Tabela 2. Variáveis de preparo da equipe perante a emergência psiquiátrica.

\begin{tabular}{ccc}
\hline Variáveis & Sim & Não \\
\hline Se sente preparado para atender pacientes em crise psicótica? & $23.7 \%$ & $76,3 \%$ \\
Já teve algum treinamento ou preparo para este tipo de atendimento? & $34,2 \%$ & $65,8 \%$ \\
Admissão de pacientes em crise psicótica interfere nas atividades laborais do trabalhador ou Equipe? & $70,27 \%$ & $29,72 \%$ \\
Já teve alguma experiência com atendimento ao paciente psiquiátrico? & $52,63 \%$ & $47,36 \%$ \\
\hline
\end{tabular}

Conforme a tabela 2, apesar da maioria dos entrevistados relatar que já estiveram mediante a um atendimento com paciente psiquiátrico, estes consideram-se como despreparados diante de uma crise psicótica, além de declarar que não tiveram nenhum treinamento especifico para atendimentos em crise psicótica. A maioria também auto avalia que este atendimento interfere nas atividades laborais do trabalhador e equipe.

Considerando as variáveis quanto ao enfrentamento dos participantes da pesquisa diante da crise psicótica, identificaram-se categorias de despreparo e tensão.

Nas categorias, destacou-se:

"Situação tensa e exige muitas habilidades."

"Para fazer medicações é uma briga, e muita das vezes não se realiza, pois pacientes em crise, é difícil de conter... foi muito difícil."

"Tive que acompanhar uma mãe que se encontrava internada na maternidade até o Eduardo Ribeiro, pois fiquei com receio dela ter crise algo desse tipo, e caso ela tivesse não ia saber como lhe dá com aquilo, ela só me olhava com cara de raiva!"

Na categoria de qualificação e preparo, destacou-se:

"O que devesse procurar é o equilíbrio no ato do atendimento."

"Os entrevistados que alegaram despreparo, mostram valorizar o preparo e treinamento como forma de solução para melhorar o atendimento perante a esse tipo de caso."

"Creio que o sistema teria que se atualizar tanto em preparar equipes tanto médica e de enfermagem para realmente tratar esses pacientes da melhor forma possivel e dar realmente uma melhor estrutura onde eles podem ser tratados de forma mais humana."

"Para uma melhor recepção ao cliente, o governo deveria disponibilizar cursos de treinamentos aos funcionários para um melhor atendimento, sendo adequado a situação. "

"Capacitar a atenção básica e disponibilizar profissionais especialistas para atendimento na Rede. Sabemos que o maior problema hoje é a falta de profissionais especialistas disponíveis que atendam a grande demanda. ”

"Fora apresentadas opiniões sobre a necessidade de ter um local apropriado e diferenciado para estes tipos de atendimento."

"Estrutura física própria e profissional adequada, treinamento de toda equipe, quantidade adequada de profissionais para prestar uma boa assistência, reconhecimento profissional e acompanhamento psicológico para os profissionais também. "

"Voltar a ter um único hospital preparado para este tipo de atendimento. "

"Ter uma área específica para esse tipo de atendimento e preparando os profissionais para atendê-los."

$\mathrm{Na}$ análise das respostas, evidenciamos a preocupação com o atendimento humanizado.

"O cliente deve ser abordado de maneira adequada e for bem acolhido na admissão que é a porta de entrada de quaisquer que for a instituição."

"Um atendimento humanizado, ter pessoas capacitadas para cuidar de pessoas com problemas psiquiátricos!"

\section{Discussão}

De acordo com os resultados desta pesquisa, podemos verificar uma falta significativa do preparo dos trabalhadores perante o atendimento emergencial para com um paciente em crise psicótica. Evidenciamos a necessidade de um treinamento em saúde mental contínuo e de qualidade, iniciado para o cuidado com o atendimento primário alçando por fim melhorias e objetivo de qualificação.

Nota-se uma grande falha diante de um atendimento emergencial a crise, no qual podemos relaciona-lo a má formação de profissionais eficazes e a falta de uma educação continuada, além de referências literárias 
sobre o tema e falta de recursos fornecidos pela instituição hospitalar onde cada entrevistado trabalha como por exemplo, cursos de reciclagem voltado a saúde mental para estes profissionais.

Neste contexto, alertamos sobre a necessidade de despertar o interesse nesses profissionais e nas instituições de saúde sobre a temática que envolve a conscientização e incentivo, priorizando a ética e a humanização no atendimento ao paciente com transtorno mental.

Esta pesquisa nos possibilitou observar e compreender os sentimentos dos trabalhadores da área da saúde diante da crise psiquiátrica, analisando que a única forma de melhorar, qualificar e ampliar seus conhecimentos sobre estas crises mudaria de forma significativa a qualidade do atendimento ao paciente em crise psiquiátrica (Quevedo et al., 2008).

O ensino e aprendizagem é um processo no qual auxilia a transformação pessoal e profissional de cada ser humano, lá podemos moldar a nossa percepção e melhorar a nossa qualidade de atendimento perante qualquer situação que venhamos receber seja ela psíquica ou não. Obter o conhecimento da teoria e por ela em prática, conduz o profissional a um atendimento satisfatório e resulta numa assistência mantendo uma estrutura adequada influenciando positivamente no cuidado recebido pelo paciente (Lima \& Guimarães, 2015).

\section{Considerações finais}

O Psicopata se refere a uma pessoa com um transtorno que tem como principal característica sua personalidade antissocial (TPA). Normalmente atinge mais homens que mulheres, podendo ser levada em consideração fatores genéticos, familiares, sociais e neurológicos. Os portadores de transtorno de personalidade antissocial possuem uma inteligência media e outros são bastantes inteligentes, costumam fazer uso de recursos verbais e buscam ser convincentes em seus argumentos (Barbos, Vasconcelos, \& Oselame, 2016).

Todo profissional deve se sentir preparado para auxiliar um paciente portador destes transtornos, atentarse aos pequenos sinais e sempre levar em consideração o histórico do paciente, o atendimento primário é de extrema importância para uma boa assistência e cuidado. Priorizar os critérios de segurança do paciente e também apresentar uma boa gestão integrada entre o processo de cuidado e reabilitação.

\section{Referências}

Ministério da Saúde, Secretária de Atenção à Saúde. 2013. Caderno de Atenção Básica, Saúde Mental. (34º ed). Brasília. Editora MS.19-176

Sales DK. 2020. Analise de preparo dos Enfermeiros para com a assistência ao paciente psicopata. Disponível em: https://www.onlinepesquisa.com/s/9ad6c4b

Ministério de Saúde. 2015. Crise e Urgência emergência em Saúde Mental. Fundamentos da Atenção à Crise e Urgência em Saúde Mental. Florianópolis, SC.

Kebbe, L. M., Rôse, L. B. R., Fiorati, R. C., \& Carretta, R. Y. D. 2014. Cuidando do familiar com transtorno mental: desafios percebidos pelos cuidadores sobre as tarefas de cuidar. Saúde em Debate, 38(102), 494505.

Kondo, É. H., Vilella, J. C., Borba, L. D. O., Paes, M. R., \& Maftum, M. A. 2011. Abordagem da equipe de enfermagem ao usuário na emergência em saúde mental em um pronto atendimento. Revista da Escola de Enfermagem da USP, 45(2), 501-507.

Costa, J. P., Jorge, M. S. B., de Lima Coutinho, M. P., Costa, E. C., \& Holanda, Í. T. A. 2016. A reforma psiquiátrica e seus desdobramentos: representações sociais dos profissionais e usuários da atenção psicossocial. Psicologia e Saber Social, 5(1), 35-45.

Lima, I. C. S., \& Guimarães, A. B. 2015. Perfil das emergências psiquiátricas atendidas em serviços de urgência e emergência hospitalar. Revista Interdisciplinar, 8(2), 181-190.

Quevedo, J. 2008. Emergências Psiquiátricas. Editora Artmed.

Minicurrículo 
Uma análise do preparo e enfrentamento da equipe de enfermagem em Manaus para com o atendimento ao paciente em crise

Ana Crisllen Monteiro Sales. Graduanda em Enfermagem pela Faculdade Estácio de Sá, Manaus, Amazonas, Brasil.

Ayrton Brandão da Silva. Graduando em Enfermagem pela Faculdade Estácio de Sá, Manaus, Amazonas, Brasil.

Diana Karen Sales da Silva. Graduanda em Enfermagem pela Faculdade Estácio de Sá, Manaus, Amazonas, Brasil.

Igor Klisman da Silva Lima. Graduando em Enfermagem pela Faculdade Estácio de Sá, Manaus, Amazonas, Brasil.

Half Adriel Simplício Araújo. Graduando em Enfermagem pela Faculdade Estácio de Sá, Manaus, Amazonas, Brasil.

Leandro Silva Pimentel. Docente do Curso de Enfermagem da Faculdade Estácio do Amazonas.

Como citar: Sales, A.C.M., Silva, A.B., Silva, D.K.D., Lima, I.K.S., Araújo, H.A.S., \& Pimentel, L.S. 2021. Uma análise do preparo e enfrentamento da equipe de enfermagem em Manaus para com o atendimento ao paciente em crise psicótica. Pubsaúde, 6, a053. DOI: https://dx.doi.org/10.31533/pubsaude6.a053

Recebido: 31 mai. 2021.

Revisado e aceito: 15 jul. 2021.

Conflito de interesse: os autores declaram, em relação aos produtos e companhias descritos nesse artigo, não ter interesses associativos, comerciais, de propriedade ou financeiros que representem conflito de interesse.

Licenciamento: Este artigo é publicado na modalidade Acesso Aberto sob a licença Creative Commons Atribuição 4.0 (CC-BY 4.0). 